\title{
Some New Types of Transitivity and Minimality
}

\section{Mohammed Nokhas Murad Kaki}

Math Department, College of Science, University of Sulaymaniyah, Sulaymaniyah, Iraqi

Email:mohammed.murad@univsul.edu.iq,muradkakaee@yahoo.com

How to cite this paper: Kaki, M.N.M. (2018) Some New Types of Transitivity and Minimality. Open Access Library Journal, 5: e4852.

https://doi.org/10.4236/oalib.1104852

Received: August 20, 2018

Accepted: November 20, 2018

Published: November 23, 2018

Copyright (C) 2018 by author and Open Access Library Inc.

This work is licensed under the Creative Commons Attribution International License (CC BY 4.0).

http://creativecommons.org/licenses/by/4.0/

\section{(c) () Open Access}

\begin{abstract}
In this paper, we introduce and study the relationship between two different notions of transitive maps, namely topological $\alpha$-transitive maps, topological $\theta$-transitive maps and investigate some of their properties in two topological spaces $\left(X, \tau^{\alpha}\right)$ and $\left(X, \tau^{\theta}\right), \tau^{\alpha}$ denotes the $\alpha$-topology (resp. $\tau^{\theta}$ denotes the $\theta$-topology) of a given topological space $(X, \tau)$. The two notions are defined by using the concepts of $\alpha$-irresolute map and $\theta$-irresolute map respectively Also, we define and study the relationship between two types of minimal mappings, namely, $\alpha$-minimal mapping and $\theta$-minimal mapping, The main results are the following propositions: 1) Every topologically $\alpha$-transitive map is transitive map, but the converse is not necessarily true. 2) Every topologically $\alpha$-minimal map is minimal map, but the converse is not necessarily true. 3) The converse of (1) and (2) is not necessarily true unless every nowhere dense set in $(X, \tau)$ is closed. 4) Also, if every $\alpha$-open set is locally closed then every transitive map implies topological $\alpha$-transitive.
\end{abstract}

\section{Subject Areas}

Mathematical Analysis

\section{Keywords}

Topologically $\theta$-Transitive, $\alpha$-Irresolute, $\theta$-Transitive, $\theta$-Dense

\section{Introduction}

The concept of topological transitivity goes back to G. D. Birkhoff who introduced it in 1920 (for flows). This article will concentrate on topological transitivity of dynamical systems given by continuous mappings in metric spaces. A dynamical system is a rule for time evolution on a state space. Intuitively, a topologically transitive dynamical system has points which eventually move under iteration from one arbitrarily small open set to any other. Consequently, such a 
dynamical system cannot be decomposed into two disjoint sets with nonempty interiors which do not interact under the transformation. Birkhoff was one of the most important leaders in American mathematics in his generation, and during his time he was considered by many to be the preeminent American mathematician.

Recently there has been some interest in the notion of a locally closed subset of a topological space. According to Bourbaki [1] a subset $S$ of a space $(X, \tau)$ is called locally closed if it is the intersection of an open set and a closed set. Ganster and Reilly used locally closed sets in [2] and [3] to define the concept of LC-continuity; i.e. a function $f:(X, \tau) \rightarrow(X, \sigma)$ is LC-continuous if the inverse with respect to $f$ of any open set in $Y$ is closed in $X$. The study of semi open sets and semi continuity in topological spaces was initiated by Levine [4]. Bhattacharya and Lahiri [5] introduced the concept of semi generalized closed sets in topological spaces analogous to generalized closed sets which were introduced by Levine [6]. Throughout this paper, the word "space" will mean topological space. The collections of semi-open, semi-closed sets and $\alpha$-sets in $(X, \tau)$ will be denoted by $S O(X, \tau), S C(X, \tau)$ and $\tau^{\alpha}$ respectively. Njastad [7] has shown that $\tau^{\alpha}$ is a topology on $X$ with the following properties: $\tau \subseteq \tau^{\alpha}$, $\left(\tau^{\alpha}\right)^{\alpha}=\tau^{\alpha}$ and $S \in \tau^{\alpha}$ if and only if $S=U \backslash N$ where $U \in \tau$ and $N$ is nowhere dense $($ i.e. $\operatorname{Int}(\mathrm{Cl}(N))=\varphi)$ in $(X, \tau)$. Hence $\tau=\tau^{\alpha}$ if and only if every nowhere dense (nwd) set in $(X, \tau)$ is closed, therefore every transitive map implies $\alpha$-transitive. Also if every $\alpha$-open set is locally closed then every transitive map implies $\alpha$-transitive; and this structure also occurs if $(X, \tau)$ is locally compact and Hausdorff ([8], p. 140, Ex. B) and every $\alpha$-open set is locally compact, then every $\alpha$-open set is locally closed. Clearly every $\alpha$-set is semi-open and every nwd set in $(X, \tau)$ is semi-closed. Andrijevic [9] has observed that $S O\left(X, \tau^{\alpha}\right)=S O(X, \tau)$, and that $N \subseteq X$ is nwd in $\left(X, \tau^{\alpha}\right)$ if and only if $\mathrm{N}$ is nwd in $(X, \tau)$.

In 1943, Fomin [10] introduced the notion of $\theta$-continuous maps. The notions of $\theta$-open sets, $\theta$-closed sets and $\theta$-closure where introduced by Velicko [11] for the purpose of studying the important class of $\mathrm{H}$-closed spaces in terms of arbitrary fiber-bases. Dickman and Porter [12] [13], Joseph [14] and Long and Herrington [15] continued the work of Velicko. We introduce the notions of $\theta$-type transitive maps, $\theta$-minimal maps and show that some of their properties are analogous to those for topologically transitive maps. Also, we give some additional properties of $\theta$-irresolute maps. We denote the interior and the closure of a subset $A$ of $X$ by $\operatorname{Int}(A)$ and $C l(A)$, respectively. By a space $X$, we mean a topological space $(X, \tau)$ A point $X \in X$ is called a $\theta$-adherent point of $A$ [11], if $A \cap C l(V) \neq \phi$ for every open set $V$ containing $x$. The set of all $\theta$-adherent points of a subset $A$ of $X$ is called the $\theta$-closure of $A$ and is denoted by $C l_{\theta}(A)$. A subset $A$ of $X$ is called $\theta$-closed if $A=C l_{\theta}(A)$. Dontchev and Maki [14] have shown that if $A$ and $B$ are subsets of a space $(X, \tau)$, then $C l_{\theta}(A \cup B)=C l_{\theta}(A) \cup C l_{\theta}(B)$ and $C l_{\theta}(A \cap B)=C l_{\theta}(A) \cap C l_{\theta}(B)$. Note al- 
so that the $\theta$-closure of a given set need not be a $\theta$-closed set. But it is always closed. Dickman and Porter [12] proved that a compact subspace of a Hausdorff space is $\theta$-closed. Moreover, they showed that a $\theta$-closed subspace of a Hausdorff space is closed. Jankovic [16] proved that a space $(X, \tau)$ is Hausdorff if and only if every compact set is $\theta$-closed. The complement of a $\theta$-closed set is called a $\theta$-open set. The family of all $\theta$-open sets forms a topology on $X$ and is denoted by $\tau^{\theta}$ or $\theta$-topology. This topology is coarser than $\tau$. In general, $C l_{\theta}(A)$ will not be the closure of $A$ with respect to $\left(X, \tau^{\theta}\right)$. It is easily seen that one always has $A \subseteq C l(A) \subseteq C l_{\delta}(A) \subseteq C l_{\theta}(A) \subseteq \bar{A}^{\theta}$ where $\bar{A}^{\theta}$ denotes the closure of $A$ with respect to $\left(X, \tau^{\theta}\right)$. It is also obvious that a set $A$ is $\theta$-closed in $(X, \tau)$ if and only if it is closed in $\left(X, \tau^{\theta}\right)$. The space $\left(X, \tau^{\theta}\right)$ is called sometimes the semi regularization of $(X, \tau)$. A function $f: X \rightarrow Y$ is closure continuous [17] ( $\theta$-continuous) at $x \in X$ if given any open set $V$ in $Y$ containing $f(x)$, there exists an open set $U$ in $X$ containing $x$ such that $f(C l(U)) \subseteq C l(V)$ [17] In this paper, we will define new classes of topological transitive maps called $\alpha$-type transitive, $\theta$-type transitive and new classes of $\alpha$-minimal maps $\theta$-minimal maps. We have shown that every $\alpha$-transitive map is a $\theta$-type transitive map, but the converse not necessarily true and that every $\alpha$-minimal map is a $\theta$-minimal map, but the converse not necessarily true we will also study some of their properties.

\section{Preliminaries and Definitions}

In this section, we recall some of the basic definitions. Let $X$ be a space and $A \subset X$. The intersection (resp. closure) of $A$ is denoted by $\operatorname{Int}(A)(\operatorname{resp} . C l(A)$.

Definition 2.1 [4] A subset $A$ of a topological space $X$ will be termed semi-open (written S.O.) if and only if there exists an open set $U$ such that $U \subset A \subset C l(U)$.

Definition 2.2 [5] Let $A$ be a subset of a space $X$ then semi closure of $A$ defined as the intersection of all semi-closed sets containing $A$ is denoted by $s C l A$.

Definition 2.3 [10] Let $(X, \tau)$ be a topological space and $\alpha$ an operator from $\tau$ to $P(X)$ i.e. $\alpha$ : $\tau \rightarrow P(X)$, where $P(X)$ is a power set of $X$. We say that $\alpha$ is an operator associated with $\tau$ if $U \subset \alpha(U)$ for all $U \in \tau$.

Definition 2.4 [18] Let $(X, \tau)$ be a topological space and $\alpha$ an operator associated with $\tau$. A subset $A$ of $X$ is said to be $\alpha$-open if for each $x \in X$ there exists an open set $U$ containing $x$ such that $\alpha(U) \subset A$. Let us denote the collection of all $\alpha$-open, semi-open sets in the topological space $(X, \tau)$ by $\tau^{\alpha}, S O(\tau)$, respectively. We then have $\tau \subseteq \tau^{\alpha} \subseteq S O(\tau)$. A subset $B$ of $X$ is said to be $\alpha$-closed [7] if its complement is $\alpha$-open.

Definition 2.5 [10] Let $(X, \tau)$ be a space. An operator $\alpha$ is said to be regular if, for every open neighborhoods $U$ and $V$ of each $x \in X$, there exists a neighborhood $W$ of $x$ such that $\alpha(W) \subset \alpha(U) \subset \alpha(V)$.

Note that the family $\tau^{\alpha}$ of $\alpha$-open sets in $(X, \tau)$ always forms a topology on $X$, when $\alpha$ is considered to be regular finer than $\tau$. 
Theorem 2.6 [20] For subsets $A, B$ of a space $X$, the following statements hold:

1) $D(A) \subset D_{\theta}(A)$ where $D(A)$ is the derived set of $A$;

2) If $A \subset B$, then $A \subset B$;

3) $D_{\theta}(A) \cup D_{\theta}(B)=D_{\theta}(A \cup B)$ and $D_{\theta}(A \cap B) \subset D_{\theta}(A) \cap D_{\theta}(B)$.

Note that the family $\tau^{\theta}$ of $\theta$-open sets in $(X, \tau)$ always forms a topology on $X$ denoted $\theta$-topology and that $\theta$-topology coarser than $\tau$.

Definition 2.7 [19] Let $A$ be a subset of a space $X$. A point $X$ is said to be an $\alpha$-limit point of $A$ if for each $\alpha$-open $U$ containing $X, U \cap(A \backslash x) \neq \phi$. The set of all $\alpha$-limit points of $A$ is called the $\alpha$-derived set of $A$ and is denoted by $D_{\alpha}(A)$.

Definition 2.8 [19] For subsets $A$ and $B$ of a space $X$, the following statements hold true:

1) $D_{\alpha}(A) \subset D(A)$ where $D(A)$ is the derived set of $A$;

2) If $A \subset B$ then $D_{\alpha}(A) \subset D_{\alpha}(B)$;

3) $D_{\alpha}(A) \cup D_{\alpha}(B) \subset D_{\alpha}(A \cup B)$;

4) $D_{\alpha}\left(A \cup D_{\alpha}(A)\right) \subset A \cup D_{\alpha}(A)$.

Definition 2.9 [18] The point $x \in X$ is in the $\alpha$-closure of a set $A \subset X$ if $\alpha(U) \cap A \neq \varphi$, for each open set $U$ containing $X$. The $\alpha$-closure of a set $A$ is the intersection of all $\alpha$-closed sets containing $A$ and is denoted by $C l_{\alpha}(A)$.

Remark 2.10 For any subset $A$ of the space $X, A \subset C l(A) \subset C l_{\alpha}(A)$.

Definition 2.11 [18] Let $(X, \tau)$ be a topological space. We say that a subset $A$ of $X$ is $\alpha$-compact if for every $\alpha$-open covering $\Pi$ of $A$ there exists a finite sub-collection $\left\{C_{1}, C_{2}, \cdots, C_{n}\right\}$ of $\Pi$ such that $A \subset \bigcup_{i=1}^{n} C_{i}$. Properties of $\alpha$-compact spaces have been investigated by Rosa, E etc. and Kasahara, S [18]. The following results were given by Rosas, $\mathrm{E}$ etc. [18].

Theorem 2.12 Let $(X, \tau)$ be a topological space and $\alpha$ an operator associated with $\tau$. $A \subset X$ and $K \subset A$. If $A$ is $\alpha$-compact and $K$ is $\alpha$-closed then $K$ is $\alpha$-compact.

Theorem 2.13 Let $(X, \tau)$ be a topological space and $\alpha$ be a regular operator on $\tau$. If $X$ is $\alpha-T_{2}$ (see Rosa, E etc. and Kasahara, S) [10] and $K \subset X$ is $\alpha$-compact then $K$ is $\alpha$-closed.

Definition 2.14 [18] The intersection of all $\alpha$-closed sets containing $A$ is called the $\alpha$-closure of $A$, denoted by $C l_{\alpha}(A)$.

Remark 2.15 For any subset $A$ of the space $X, A \subset C l(A) \subset C l_{\alpha}(A)$.

Lemma 2.16 For subsets $A$ and $A_{i} \quad(i \in I)$ of a space $(X, \tau)$, the following hold:

1) $A \subset C l_{\alpha}(A)$;

2) $C l_{\alpha}(A)$ is closed; $C l_{\alpha}\left(C l_{\alpha}(A)\right)=C l_{\alpha}(A)$;

3) If $A \subset B$ then $C l_{\alpha}(A) \subset C l_{\alpha}(B)$;

4) $C l_{\alpha}\left(\cap\left(A_{i}: i \in I\right)\right) \subset \cap\left(C l_{\alpha}(A): i \in I\right)$;

5) $C l_{\alpha}\left(\cup\left(A_{i}: i \in I\right)\right)=\cup\left(C l_{\alpha}(A): i \in I\right)$.

Lemma 2.17 The collection of $\alpha$-compact subsets of $X$ is closed under finite 
unions. If $\alpha$ is a regular operator and $X$ is an $\alpha-T_{2}$ space then it is closed under arbitrary intersection.

Definition 2.18 Let $(X, \tau)$ be a topological any space, $A$ subset of $X$, The $\operatorname{int}_{\alpha}(A)=\cup\{U: U$ is $\alpha$-open and $U \subset A\}$.

Remark 2.19 A subset $A$ is $\alpha$-open if and only if $\operatorname{int}_{\alpha}(A)=A$.

Proof: The proof is obvious from the definition.

Definition 2.20 Let $(X, \tau)$ and $(Y, \sigma)$ be two topological spaces, a map $f: X \rightarrow Y$ is said to be $\alpha$-continuous if for each open set $H$ of $Y, f^{-1}(H)$ is $\alpha$-open in $X$.

Theorem 2.21 [19] For any subset $A$ of a space $X, C l_{\alpha}(A)=A \cup C l_{\alpha}(A)$.

Theorem 2.22 [19] For subsets $A, B$ of a space $X$, the following statements are true:

1) $\operatorname{int}_{\alpha}(A)$ is the largest $\alpha$-open contained in $A$;

2) $\operatorname{int}_{\alpha}\left(\operatorname{int}_{\alpha}(A)\right)=\operatorname{int}_{\alpha}(A)$;

3) If $A \subset B$ then $\operatorname{int}_{\alpha}(A) \subset \operatorname{int}_{\alpha}(B)$;

4) $\operatorname{int}_{\alpha}(A) \cup \operatorname{int}_{\alpha}(B) \subset \operatorname{int}_{\alpha}(A \cup B)$;

5) $\operatorname{int}_{\alpha}(A) \cap \operatorname{int}_{\alpha}(B) \supset \operatorname{int}_{\alpha}(A \cap B)$.

Lemma 2.23 [7] For any $\alpha$-open set $A$ and any $\alpha$-closed set $C$, we have:

1) $C l_{\alpha}(A)=C l(A)$;

2) $\operatorname{int}_{\alpha}(C)=\operatorname{int}(C)$;

3) $\operatorname{int}_{\alpha}\left(C l_{\alpha}(A)\right)=\operatorname{int}(C l(A))$.

Remark 2.24 [19] It is not always true that every $\alpha$-open set is an open set, as shown in the following example:

Example 2.25 Let $X=\{a, b, c, d\}$ with topology $\tau=\{\phi,\{a, b\}, X\}$. Hence $\alpha(\tau)=\{\phi,\{c, d\},\{b, c, d\},\{a, c, d\}, X\}$.

So $\{b, c, d\}$ is $\alpha$-open but not open.

Theorem 2.26 [20] For subsets $A, B$ of a topological space $X$, the following statements are true:

1) $\operatorname{Int}_{\theta}(A)$ is the union of all open sets of $X$ whose closures are contained in A.

2) $A$ is $\theta$-open $\Leftrightarrow \operatorname{Int}_{\theta}(A)=A$.

3) $\operatorname{Int}_{\theta}\left(\operatorname{Int}_{\theta}(A)\right) \subset \operatorname{Int}_{\theta}(A)$.

4) $X \backslash \operatorname{Int}_{\theta}(A)=C l_{\theta}(X \backslash A)$.

5) $X \backslash C l_{\theta}(A)=\operatorname{Int}_{\theta}(X \backslash A)$.

6) If $A \subset B$ then $\operatorname{Int}_{\theta}(A) \subset \operatorname{Int}_{\theta}(B)$.

7) $\operatorname{Int}_{\theta}(A) \cup \operatorname{Int}_{\theta}(B) \subset \operatorname{Int}_{\theta}(A \cup B)$.

8) $\operatorname{Int}_{\theta}(A) \cap \operatorname{Int}_{\theta}(B)=\operatorname{Int}_{\theta}(A \cap B)$.

\section{Transitive and Minimal Systems}

Topological transitivity is a global characteristic of dynamical systems. By a dynamical system $(X, f)$ [21] we mean a topological space $X$ together with a continuous map $f: X \rightarrow X$. The space $X$ is sometimes called the phase space of the system. A set $A \subseteq X$ is called $f$-inveriant if $f(A) \subseteq A$. 
A dynamical system $(X, f)$ is called minimal if $X$ does not contain any non-empty, proper, closed $f$-inveriant subset. In such a case we also say that the map $f$ itself is minimal. Thus, one cannot simplify the study of the dynamics of a minimal system by finding its nontrivial closed subsystems and studying first the dynamics restricted to them.

Given a point $x$ in a system $(X, f), O_{f}(x)=\left\{x, f(x), f^{2}(x), \cdots\right\}$ denotes its orbit (by an orbit we mean a forward orbit even if $f$ is a homeomorphism) and $\omega_{f}(x)$ denotes its $\omega$-limit set, i.e. the set of limit points of the sequence $x, f(x), f^{2}(x), \cdots$. The following conditions are equivalent:

- $(X, f)$ is minimal,

- every orbit is dense in $X$,

- $\omega_{f}(x)=X$ for every $x \in X$.

A minimal map $f$ is necessarily surjective if $X$ is assumed to be Hausdorff and compact.

Now, we will study the Existence of minimal sets. Given a dynamical system $(X, f)$, a set $A \subseteq X$ is called a minimal set if it is non-empty, closed and invariant and if no proper subset of $A$ has these three properties. So, $A \subseteq X$ is a minimal set if and only if $(A, f \mid A)$ is a minimal system. A system $(X, f)$ is minimal if and only if $X$ is a minimal set in $(X, f)$.

The basic fact discovered by G. D. Birkhoff is that in any compact system $(X, f)$ there are minimal sets. This follows immediately from the Zorn's lemma. Since any orbit closure is invariant, we get that any compact orbit closure contains a minimal set. This is how compact minimal sets may appear in non-compact spaces. Two minimal sets in $(X, f)$ either are disjoint or coincide. A minimal set $A$ is strongly f-inveriant, i.e. $f(A)=A$. Provided it is compact Hausdorff.

Let $(X, f)$ be a topological system, and $f: X \rightarrow X \quad \alpha$ r-homeomorphism of $X$ onto itself. For $A$ and $B$ subsets of $X$, we let

$N(A, B)=\left\{n \in \mathbf{Z}: f^{n}(A) \cap B \neq \phi\right\}$.

We write $N(A, B)=N(x, B)$ for a singleton $A=\{x\}$ thus $N(x, B)=\left\{n \in \mathbf{Z}: f^{n}(x) \in B\right\}$.

For a point $x \in X$ we write $O_{f}(x)=\left\{f^{n}(x): n \in \mathbf{Z}\right\}$ for the orbit of $x$ and $C l_{\alpha}\left(O_{f}(x)\right)$ for the $\alpha$-closure of $O_{f}(x)$. We say that the topological system $(X, f)$ is $\alpha$-type point transitive if there is a point $x \in X$ with $O_{f}(x)$ $\alpha$-dense. Such a point is called $\alpha$-type transitive. We say that the topological system $(X, f)$ is topologically $\alpha$-type transitive (or just $\alpha$-type transitive) if the set $N(U, V)$ is nonempty for every pair $U$ and $V$ of nonempty $\alpha$-open subsets of $X$.

\section{Topologically $\alpha$-Transitive Maps}

In [22], we introduced and defined a new class of transitive maps that are called topologically $\alpha$-transitive maps on a topological space $(X, \tau)$, and we studied some of their properties and proved some results associated with these new definitions. We also defined and introduced a new class of $\alpha$-minimal maps. In this 
paper we discuss the relationship between topologically $\alpha$-transitive maps and $\theta$-transitive maps. On the other hand, we discuss the relationship between $\alpha$-minimal and $\theta$-minimal in dynamical systems.

Definition 3.1.1 Let $(X, \tau)$ be a topological space. A subset $A$ of $X$ is called $\alpha$-dense in $X$ if $C l_{\alpha}(A)=X$.

Remark 3.1.2 Any $\alpha$-dense subset in $X$ intersects any $\alpha$-open set in $X$.

Proof: Let $A$ be an $\alpha$-dense subset in $X$, then by definition, $C l_{\alpha}(A)=X$, and let $U$ be a non-empty $\alpha$-open set in $X$. Suppose that $A \cap U=\phi$. Therefore $B=U^{c}$ is $\alpha$-closed and $A \subset U^{c}=B$. So $C l_{\alpha}(A) \subset C l_{\alpha}(B)$, i.e. $C l_{\alpha}(A) \subset B$, but $C l_{\alpha}(A)=X$, so $X \subset B$, this contradicts that $U \neq \varphi$.

Definition 3.1.3 [23] A map $f: X \rightarrow Y$ is called $\alpha$-irresolute if for every $\alpha$-open set $H$ of $Y, f^{-1}(H)$ is $\alpha$-open in $X$.

Example 3.1.4 [22] Let $(X, \tau)$ be a topological space such that $X=\{a, b, c, d\}$ and $\tau=\{\phi, X,\{a, b\},\{b\}\}$. We have the set of all $\alpha$-open sets is $\alpha(X, \tau)=\{\phi, X,\{b\},\{a, b\},\{b, c\},\{b, d\},\{a, b, c\},\{a, b, d\}\}$ and the set of all $\alpha$-closed sets is $\alpha C(X, \tau)=\{\phi, X,\{c, d\},\{a, c, d\},\{a, d\},\{a, c\},\{d\},\{c\}\}$. Then define the map $f: X \rightarrow X$ as follows $f(a)=a, f(b)=b, f(c)=d$, $f(d)=c$, we have $f$ is $\alpha$-irresolute because $\{b\}$ is $\alpha$-open and $f^{-1}(\{b\})=\{b\}$ is $\alpha$-open; $\{a, b\}$ is $\alpha$-open and $f^{-1}(\{a, b\})=\{a, b\}$ is $\alpha$-open; $\{b, c\}$ is $\alpha$-open and $f^{-1}(\{b, c\})=\{b, d\}$ is $\alpha$-open; $\{a, b, c\}$ is $\alpha$-open and $f^{-1}(\{a, b, c\})=\{a, b, d\}$ is $\alpha$-open; $\{a, b, d\}$ is $\alpha$-open and $f^{-1}(\{a, b, d\})=\{a, b, c\}$ is $\alpha$-open so $f$ is $\alpha$-irresolute.

Definition 3.1.5 A subset $A$ of a topological space $(X, \tau)$ is said to be nowhere $\alpha$-dense, if its $\alpha$-closure has an empty $\alpha$-interior, that is, $\operatorname{int}_{\alpha}\left(C l_{\alpha}(A)\right)=\phi$.

Definition 3.1.6 [22] Let $(X, \tau)$ be a topological space, $f: X \rightarrow X$ be $\alpha$-irresolute map then $f$ is said to be topological $\alpha$-transitive if every pair of non-empty $\alpha$-open sets $U$ and $V$ in $X$ there is a positive integer $n$ such that $f^{n}(U) \cap V \neq \phi$. In the forgoing example 3.1.4: we have $f$ is $\alpha$-transitive because $b$ belongs to any non-empty $\alpha$-open set $V$ and also belongs to $f(U)$ for any $\alpha$-open set it means that $f(U) \cap V \neq \phi$ so $f$ is. $\alpha$-transitive.

Example 3.1.7 Let $(X, \tau)$ be a topological space such that $X=\{a, b, c\}$ and $\tau=\{\phi,\{a\}, X\}$. Then the set of all $\alpha$-open sets is $\tau^{\alpha}=\{\phi,\{a\},\{a, b\},\{a, c\}, X\}$. Define $f: X \rightarrow X$ as follows $f(a)=b, f(b)=b, f(c)=c$. Clearly $f$ is continuous because $\{a\}$ is open and $f(\{a\})=\phi$ is open. Note that $f$ is transitive because $f(\{a\})=\{b\}$ implies that $f(\{a\}) \cap\{b\} \neq \phi$. But $f$ is not $\alpha$-transitive because for each $n$ in $N, f^{n}(\{a\}) \cap\{a, c\}=\phi$; since $f^{n}(\{a\})=\{b\}$ for every $n \in N$, and $\{b\} \cap\{a, c\}=\phi$. So we have $f$ is not $\alpha$-transitive, so we show that transitivity not implies $\alpha$-transitivity.

Definition 3.1.8 Let $(X, \tau)$ be a topological space. A subset $A$ of $X$ is called $\theta$-dense in $X$ if $C l_{\theta}(A)=X$.

Remark 3.1.9 Any $\theta$-dense subset in $X$ intersects any $\theta$-open set in $X$.

Proof: Let $A$ be a $\theta$-dense subset in $X$, then by definition, $C l_{\theta}(A)=X$, and let $U$ be a non-empty $\theta$-open set in $X$. Suppose that $A \cap U=\phi$. Therefore 
$B=U^{c}$ is $\theta$-closed because $B$ is the complement of $\theta$-open and $A \subset U^{c}=B$. So $C l_{\theta}(A) \subset C l_{\theta}(B)$, i.e. $C l_{\theta}(A) \subset B$, but $C l_{\theta}(A)=X$, so $X \subset B$, this contradicts that $U \neq \varphi$.

Definition 3.1.10 [24] A function $f: X \rightarrow X$ is called $\theta$-irresolute if the inverse image of each $\theta$-open set is a $\theta$-open set in $X$.

Definition 3.1.11 A subset $A$ of a topological space $(X, \tau)$ is said to be nowhere $\theta$-dense, if its $\theta$-closure has an empty $\theta$-interior, that is, $\operatorname{int}_{\theta}\left(C l_{\theta}(A)\right)=\phi$.

Definition 3.1.12 [25] Let $(X, \tau)$ be a topological space, and $f: X \rightarrow X$ $\theta$.irresolute) map, then $f$ is said to be topologically $\theta$-type transitive map if for every pair of $\theta$-open sets $U$ and $V$ in $X$ there is a positive integer $n$ such that $f^{n}(U) \cap V \neq \phi$.

Theorem 3.1.13 every theta-type transitive map implies transitive nap if ( $X$, $\tau)$ is regular.

Note that a space $(X, \tau)$ is regular if and only if $\tau=\tau^{\theta} \quad$ [26].

Theorem 3.1.14 [22] Let $(X, \tau)$ be a topological space and $f: X \rightarrow X$ be $\alpha$-irresolute map. Then the following statements are equivalent:

1) $f$ is topological $\alpha$-transitive map.

2) For every nonempty $\alpha$-open set $U$ in $X, \bigcup_{n=0}^{\infty} f^{n}(U)$ is $\alpha$-dense in $X$.

3) For every nonempty $\alpha$-open set $U$ in $X, \bigcup_{n=0}^{\infty} f^{-n}(U)$ is $\alpha$-dense in $X$.

4) If $B \subset X$ is $\alpha$-closed and $B$ is $f$-invariant i.e. $f(B) \subset B$ then $B=X$ or $\mathrm{B}$ is nowhere $\alpha$-dense.

5) If $U$ is $\alpha$-open and $f^{-1}(U) \subset U$ then $U$ is either empty set or $\alpha$-dense in $X$.

Theorem 3.1.15 [25] Let $(X, \tau)$ be a topological space and $f: X \rightarrow X$ be $\theta$-irresolute map. Then the following statements are equivalent:

1) $f$ is $\theta$-type transitive map.

2) $\bigcup_{n=0}^{\infty} f^{n}(D)$ is $\theta$-dense in $X$, with $D$ is $\theta$-open set in $X$.

3) $\bigcup_{n=0}^{\infty} f^{-n}(D)$ is $\theta$-dense in $X$ with $D$ is $\theta$-open set in $X$.

4) If $B \subset X$ is $\theta$-closed and $f(B) \subset B$ then $B=X$ or $B$ is nowhere $\theta$-dense.

5) If $f^{-1}(D) \subset D$ and $D$ is $\theta$-open in $X$ then $D=\phi$ or $D$ is $\theta$-dense in $X$, we have to prove th theorem.

\section{4. $\alpha$-Minimal Functions}

We introduced a new definition on $\alpha$-minimal [22] (resp. $\theta$-minimal [25]) maps and we studied some new theorems associated with these definitions.

Given a topological space $X$, we ask whether there exists $\alpha$-irresolute (resp. $\theta$-irresolute) map on $X$ such that the set $\left\{f^{n}(x): n \geq 0\right\}$, called the orbit of $X$ and denoted by $O_{f}(x)$, is $\alpha$-dense(resp. $\theta$-dense) in $X$ for each $x \in X$. A partial 
answer will be given in this section. Let us begin with a new definition.

Definition 4.1 ( $\alpha$-minimal) Let $X$ be a topological space and $f$ be $\alpha$-irresolute map on $X$ with $\alpha$-regular operator associated with the topology on $X$. Then the dynamical system $(X, f)$ is called $\alpha$-minimal system (or $f$ is called $\alpha$-minimal map on $X$ ) if one of the three equivalent conditions [22] hold:

1) The orbit of each point of $X$ is $\alpha$-dense in $X$.

2) $C_{\alpha}\left(O_{f}(x)\right)=X$ for each $x \in X$.

3) Given $x \in X$ and a nonempty $\alpha$-open $U$ in $X$, there exists $n \in N$ such that $f^{n}(x) \in U$.

Theorem 4.2 [22] For $(X, f)$ the following statements are equivalent:

1) $f$ is an $\alpha$-minimal map.

2) If $E$ is an $\alpha$-closed subset of $X$ with $f(E) \subset E$, we say $E$ is invariant. Then $E=\phi$ or $E=X$.

3) If $U$ is a nonempty $\alpha$-open subset of $X$, then $\bigcup_{n=0}^{\infty} f^{-n}(U)=X$.

\section{Conclusions}

We have following propositions:

1) Every topologically $\alpha$-transitive map is transitive map, but the converse is not necessarily true.

2) Every topologically $\alpha$-minimal map is minimal map, but the converse is not necessarily true.

3) The converse of (1) and (2) is not necessarily true unless every nowhere dense set in $(X, \tau)$ is closed.

Also, if every $\alpha$-open set is locally closed then every transitive map implies topological $\alpha$-transitive.

\section{Conflicts of Interest}

The author declares no conflicts of interest regarding the publication of this paper.

\section{References}

[1] Bourbaki, N. (1966) General Topology Part 1, Addison Wesley, Reading, MA.

[2] Ganster, M. and Reilly, I.L. (1990) A Decomposition of Continuity. Acta Mathematica Hungarica, 56, 299-301. https://doi.org/10.1007/BF01903846

[3] Ganster, M. and Reilly, I.L. (1989) Locally Closed Sets and LC-Continuous Functions. International Journal of Mathematics and Mathematical Sciences, 12, 417-424. https://doi.org/10.1155/S0161171289000505

[4] Levine, N. (1963) Semi-Open Sets and Semi-Continuity in Topological Spaces. The American Mathematical Monthly, 70, 36-41. https://doi.org/10.1080/00029890.1963.11990039

[5] Bhattacharya, P. and Lahiri, K.B. (1987) Semi-Generalized Closed Sets in Topology. Indian Journal of Mathematics, 29, 376-382.

[6] Levine, N. (1970) Generalized Closed Sets in Topology. Rendiconti del Circolo 
Matematico di Palermo, 19 , 89-96.

[7] Ogata, N. (1965) On Some Classes of Nearly Open Sets. Pacific Journal of Mathematics, 15, 961-970. https://doi.org/10.2140/pjm.1965.15.961

[8] Engelking, R. (1968) Outline of General Topology. North Holland Publishing Company, Amsterdam.

[9] Andrijević, D. (1994) Some Properties of the Topology of $\alpha$-Sets. Matematički Vesnik, 36, 1-9.

[10] Rosas, E. and Vielina, J. (1998) Operator-Compact and Operator-Connected Spaces. Scientific Mathematica, 1, 203-208.

[11] Velicko, N.V. (1966) H-Closed Topological Spaces. American Mathematical Society Translations, 78, 102-118.

[12] Dickman, R.F. and Porter, J.R. (1975) $\theta$-Closed Subsets of Hausdorff Spaces. Pacific Journal of Mathematics, 59, 407-415. https://doi.org/10.2140/pjm.1975.59.407

[13] Dickman Jr., R.F. and Porter, J.R. (1977) $\theta$-Perfect and $\theta$-Absolutely Closed Functions. Illinois Journal of Mathematics, 21, 42-60.

[14] Dontchev, J. and Maki, H. (1998) Groups of $\theta$-Generalized Homeomorphisms and the Digital Line. Topology and Its Applications, 20, 1-16.

[15] Long, P.E. and Herrington, L.L. (1982) The $\tau_{\theta}$-Topology and Faintly Continuous Functions. Kyungpook Mathematical Journal, 22, 7-14.

[16] Jankovic, D.S. (1986) $\theta$-Regular Spaces. International Journal of Mathematics and Mathematical Sciences, 8, 615-619. https://doi.org/10.1155/S0161171285000667

[17] Saleh, M. (2003) On $\theta$-Continuity and Strong $\theta$-Continuity. Applied Mathematics E-Notes, 3, 42-48.

[18] Kasahara, S. (1979) Operation-Compact Spaces. Mathematica Japonica, 24, 97-105.

[19] Caldas, M. (2003) A Note on Some Applications of $\alpha$-Open Sets. UMMS, 2, 125-130.

[20] Caldas, M., Jafari, S. and Kovar, M.M. (2004) Some Properties of $\theta$-Open Sets. Divulgaciones Matematicas, 12, 161-169.

[21] http://www.scholarpedia.org/article/Minimal_dynamical_systems

[22] Kaki, M.N.M. (2012) Topologically $\alpha$ Transitive Maps and Minimal Systems. General Mathematics Notes, 10, 43-53.

[23] Maheshwari, N.S. and Thakur, S.S. (1980) On $\alpha$-Irresolute Mappings. Tamkang Journal of Mathematics, 11, 209-214.

[24] Khedr, F.H. and Noiri, T. (1986) On $\theta$-Irresolute Functions. Indian Journal of Mathematics, 28, 211-217.

[25] Kaki, M.N.M. (2012) Introduction to $\theta$-Type Transitive Maps on Topological Spaces. International Journal of Basic \& Applied Sciences, 12, 104-108.

[26] Joseph, J.E. (1979) $\theta$-Closure and $\theta$-Subclosed Graphs. The Mathematical Chronicle, 8, 99-117. 